\title{
1 Central Role of Cognitive Control Networks in Weight Loss During Voluntary Calorie
}

\section{Restriction}

3 Selin Neseliler ${ }^{1}$, Wen $\mathrm{Hu}^{2}$, Kevin Larcher $^{1}$, Maria Zacchia ${ }^{1}$, Mahsa Dadar ${ }^{1}$, Stephanie G.

4 Scala ${ }^{1}$, Marie Lamarche ${ }^{2}$, Yashar Zeighami ${ }^{1}$, Stephen C. Stotland ${ }^{3}$, Maurice Larocque ${ }^{4}$, Errol B.

5 Marliss $^{2}$, and Alain Dagher*

6 1. Montreal Neurological Institute, McGill University, Montréal, Canada

7 2. Crabtree Nutrition Laboratories, Department of Medicine, McGill University Health Centre

8 Research Institute, McGill University, Montréal, Canada

9 3. Department of Psychology, McGill University, Montréal, Canada

10 4. Clinique Motivation Minceur, Montréal, Canada

11 * Lead Contact

13 Word count of summary: 149

$14 \quad$ Figures and Tables: 5 Figures

15 Supplemental data: 9 Tables, 1 Figure

16

17 Address for correspondence:

18 Alain Dagher, MD

19 Montreal Neurological Institute

203801 University St., Montréal QC Canada H3A 2B4

21 email: alain.dagher@mcgill.ca 


\section{Summary}

23 Insufficient responses to hypocaloric diets have been attributed to hormonal adaptations that

24 override self-control of food intake. We tested this hypothesis by measuring brain fMRI reactivity

25 to food cues and circulating energy-balance hormones in 24 overweight/obese participants before,

26 and 1 and 3 months after starting a calorie restriction diet. Increased activity in prefrontal regions at

27 month 1 correlated with weight loss at months 1 and 3 . Weight loss was also correlated with

28 increased plasma ghrelin and decreased leptin at month 1, and these changes were associated with

29 greater food cue reactivity in reward-related brain regions. However, the reduction in leptin did not

30 counteract weight loss; indeed, it was correlated with further weight loss at month 3 . Activation in a

31 network of prefrontal regions associated with self-control could contribute to individual differences

32 in weight loss and maintenance, whereas we failed to find that the hormonal adaptations play a

33 major role. 


\section{Introduction}

42 Weight loss can improve comorbidities and cardiometabolic risk factors associated with obesity.

43 Two-thirds of the American population have undertaken reducing diets at least once (Gudzune et

44 al., 2015). However, achieving and maintaining weight loss remain challenging (Anastasiou et al.,

45 2015). Several studies indicate that high-order executive cognitive processes implicated in self-

46 regulation play an important role in healthy food decisions and weight management (Gettens and

47 Gorin, 2017; Michaud et al., 2017; Stoeckel et al., 2017). However, hormonal responses to

48 negative energy during calorie restriction balance can modulate the activity of brain systems

49 implicated in feeding in favor of increased calorie intake (Berthoud et al., 2012). In humans, it

50 remains to be tested if the changes in energy balance signals during calorie restriction can modulate

51 the brain networks associated with food intake, override self-control, and oppose weight loss.

52 Brain circuitry underlying food decisions can be divided into three interacting systems: (1) a

53 homeostatic system centered around the hypothalamus; (2) a reward-related appetitive network

54 including the striatum and the ventromedial prefrontal cortex (vmPFC) that encodes the subjective

55 value of food cues; (3) an executive control network that relies on the function of interconnected

56 prefrontal regions including the anterior cingulate cortex (ACC), dorsolateral prefrontal cortex

57 (dlPFC), inferior frontal gyrus (IFG), and posterior parietal (PP) cortex (Dagher, 2012; Ochner et

58 al., 2013). Cognitive control, defined here as the ability to restrict calorie intake and to sustain

59 weight maintenance, is thought to rely on these executive structures. It is proposed that cognitive

60 control ability mediates the relationship between weight loss intention and action (Gettens and

61 Gorin, 2017). The dlPFC and IFG have been repeatedly implicated in dietary self-control, studied 
62 with functional magnetic resonance imaging (fMRI) utilizing blood-oxygen-level-dependent

63 (BOLD) contrast. Activation of the dIPFC and IFG is seen when subjects are asked to voluntarily

64 suppress the desire to eat in response to food cues (Batterink et al., 2010; Hollmann et al., 2012),

65 and predicts subsequent reduced food intake outside the lab (Lopez et al., 2014, 2017). FMRI

66 studies also support a model according to which dIPFC and IFG downregulate the activity of value-

67 encoding regions (e.g. vmPFC) when participants choose healthy over unhealthy foods or regulate

68 their food cravings (Hare et al., 2009, 2011). The relative balance of activity in regions associated

69 with self-regulation over those associated with reward has been used to compute a brain-derived

70 measure of self-regulation ability, which relates to healthier real-life food choices in dieters and

71 non-dieters (Lopez et al., 2014, 2017). This has led to dual systems theories where behavioral

72 outcomes depend on the balance between self-control and reactivity to reward. Although few

73 studies have examined brain activity longitudinally in individuals undergoing calorie restriction,

74 there is some support for the role of dlPFC in successful weight loss (Weygandt et al., 2013) and of

75 ventral striatum activity in worse outcomes (Murdaugh et al., 2012).

76 According to the dual systems theory, the magnitude of weight loss during calorie restriction will be

77 related to the following fMRI findings: (1) increased brain activity in regions associated with

78 cognitive control, (2) increased connectivity of these areas to regions ascribed to value processing

79 (e.g., vmPFC and striatum), and (3) downregulation of activity in these value-related brain regions.

80 However, central nervous system networks are also modulated by internal states, such as current

81 energy balance status (Neseliler et al., 2017; Rangel, 2013). During calorie restriction, ghrelin and

82 leptin reflect changes in energy balance. Leptin plasma levels decline rapidly in response to calorie 
83 restriction, and more slowly with reduction of fat mass (Friedman and Mantzoros, 2015). Patients

84 with leptin deficient states show increased food cue reactivity in the striatum and orbitofrontal

85 cortex (OFC) compared with controls (Aotani et al., 2012). Striatal BOLD response to food cues

86 in the striatum is reduced by leptin administration in these patients (Aotani et al., 2012; Farooqi et

87 al., 2007). In normoleptinemic participants, leptin levels negatively correlate with food cue

88 reactivity in the striatum (Grosshans et al., 2012) and leptin administration to weight-reduced

89 subjects results in increased activity in regions associated with cognitive control (Rosenbaum et al.,

90 2008). These studies suggest that reductions in leptin levels can result in increased activity in the

91 mesolimbic reward system and reduced activity in brain regions associated with cognitive control.

92 Conversely, ghrelin - an orexigenic hormone secreted by the stomach - increases rapidly in

93 response to calorie deficit (Borer et al., 2009). Post-translational modification converts ghrelin to

94 acyl- ghrelin, its active form (Müller et al., 2015). Ghrelin can increase the neural response to food

95 cues in regions associated with value and motivation and potentiate food intake (Goldstone et al.,

96 2014; Malik et al., 2008). During weight loss, the fall in leptin and rise in ghrelin levels could

97 modulate the activity of brain networks involved in reward signaling to shift the balance towards

98 increased food intake (Berthoud et al., 2012).

99 We designed this study to test these two predictions on the role of the central nervous system in

100 voluntary calorie restriction: (1) that activation of cognitive control networks during fMRI would

101 predict weight loss and (2) that early weight loss should lead to changes in energy-balance signaling

102 (leptin and ghrelin), which would lead to increased activity in regions associated with reward and

103 counteract self-control mechanisms. Twenty-four individuals underwent a three-month calorie 
104 restriction program with measurement of fMRI and metabolic variables at baseline (before

105 initiating the diet), and at months 1 and 3. Body weight was also obtained at two years. During

106 fMRI they viewed 216 pictures of appetizing foods or scenery, and rated them on a 4-point scale.

107 Our results support the first prediction but not the second.

\section{Results \& Discussion}

\section{Calorie restriction resulted in weight loss}

112 The 24 participants (1 Male) had a mean age of $37.2(\mathrm{SD}= \pm 8.4)$ and a mean body mass index

113 (BMI) at entry of $30.4(\mathrm{SD}= \pm 3.2)$. The personality measures of the study population are listed

114 in Table S1. We analyzed the changes in weight, physical activity, hunger levels and energy-

115 balance hormone levels at months 1 and 3 during calorie restriction, compared with baseline

116 (Fig. 2). All the analyses were conducted using linear mixed effect modeling. Significant

117 reductions in $\mathrm{BMI}$ occurred across the three sessions of calorie restriction $(\mathrm{F}(1,62)=86.2, \mathrm{p}=$

$\left.1182.4 * 10^{-13}\right)$. Pairwise comparisons showed the reductions in BMI were significant from baseline

119 to month $1\left(\mathrm{~F}(1,61)=22.91, \mathrm{p}=1.12 * 10^{-5}\right.$, mean weight loss $\left.2.20 \pm 4.22 \mathrm{~kg}\right)$, and from month

1201 to month $3(\mathrm{~F}(1,61)=17.16, \mathrm{p}=0.0001$, mean weight loss $1.88 \pm 1.55 \mathrm{~kg})($ Fig. 2 -A, Table

121 S2). Self-reported physical activity levels across the sessions did not show significant differences

$122(\mathrm{~F}(1,31)=0.31, \mathrm{p}=0.71$, Table $\mathrm{S} 2)$. There were no significant differences in hunger assessed by

123 VAS across the sessions (Table S3). 


\section{SESSIONS}

\section{Baseline}

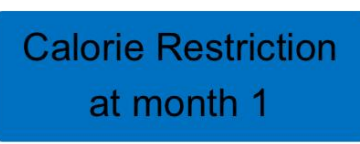

at month 1

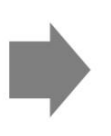

Calorie Restriction

at month 3

\section{EACH SESSION}

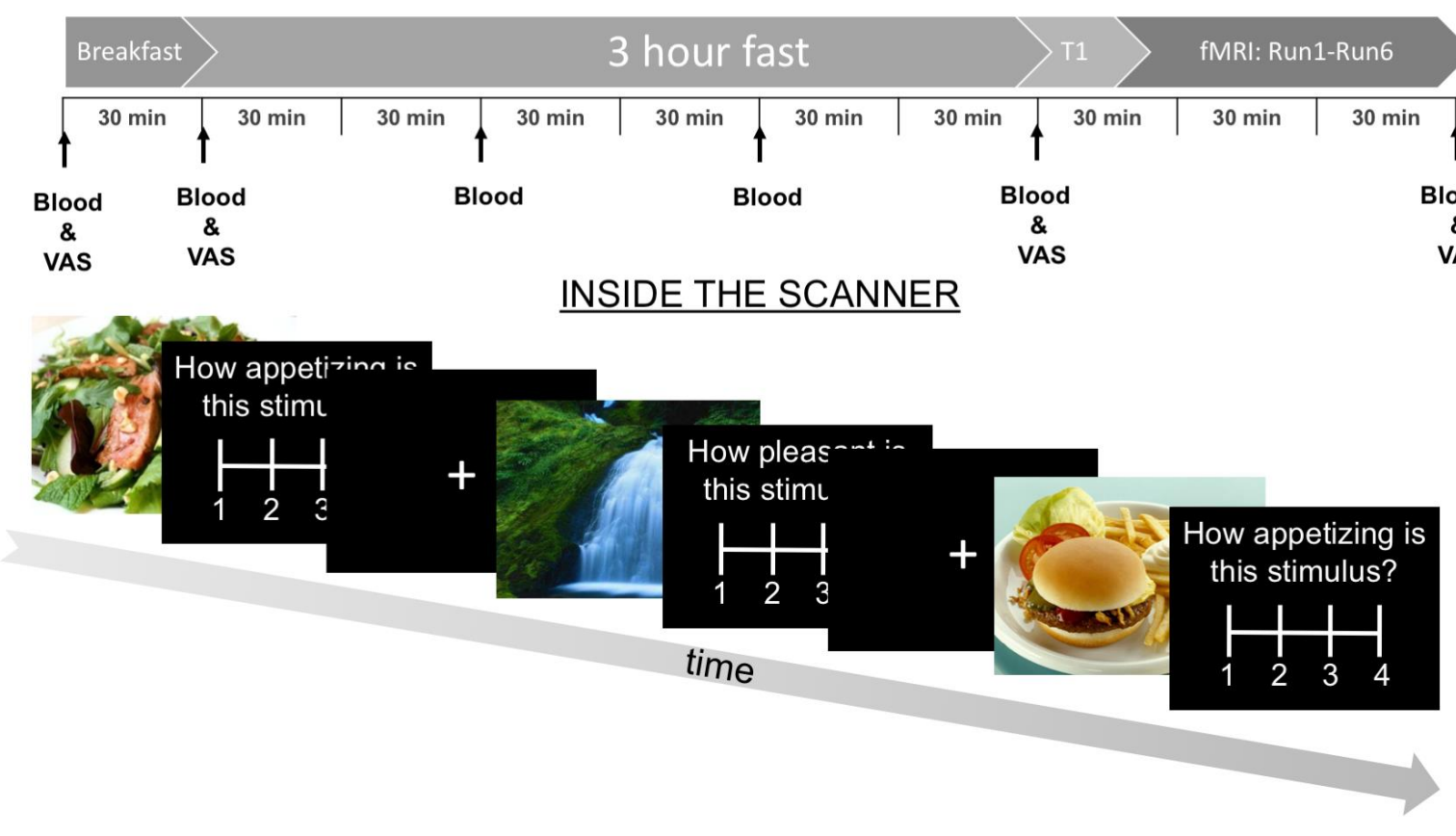

128 Figure 1. Experimental Design.

129 Participants $(\mathrm{n}=24)$ were tested three times: at baseline, then at 1 and 3 months during voluntary

130 calorie restriction. On each scan day, participants ate a standard breakfast three hours prior to

131 fMRI imaging. Venous blood and self-reported hunger level on a visual analog scale (VAS) were

132 sampled at the times indicated. Anatomical scanning (T1) was followed by six seven-minute

133 functional runs. During fMRI participants viewed pictures of foods and scenery followed by a

134 rating. 
A

C
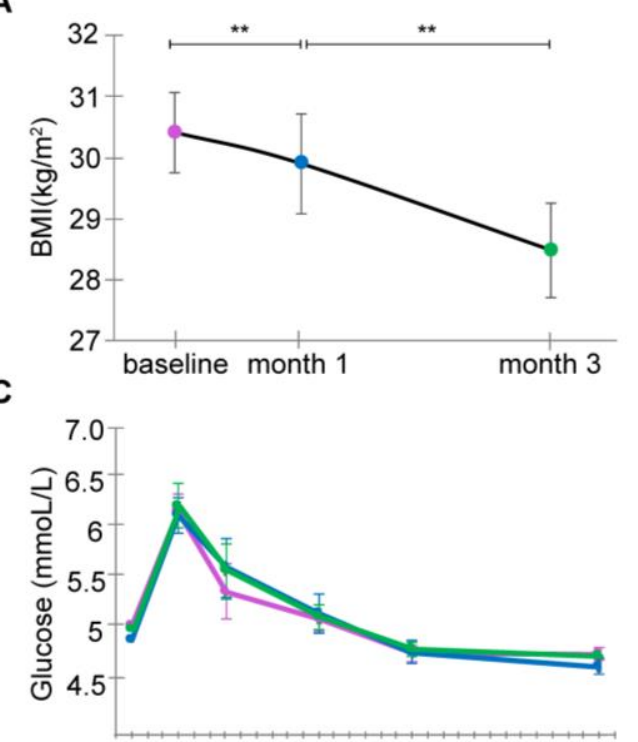

E

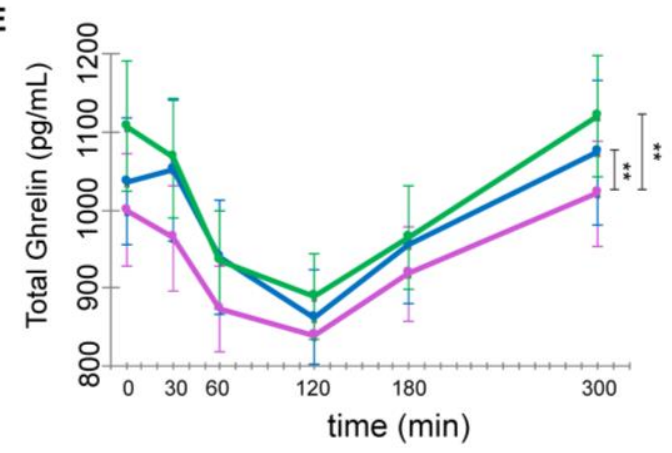

B

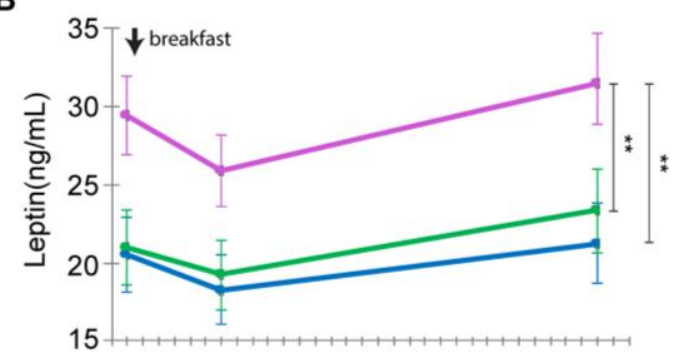

D

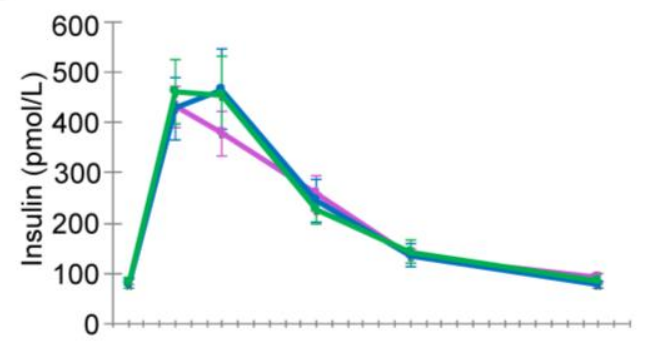

$\mathbf{F}$

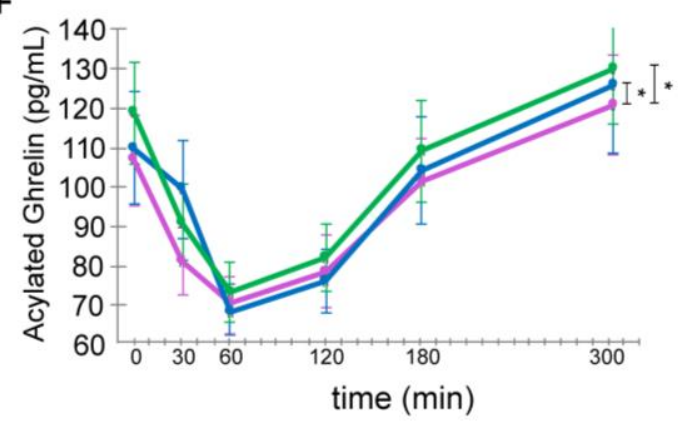

- baseline month 1 month 3

\section{Figure 2. Effects of voluntary calorie restriction.}

137 (A) BMI decreased significantly across the sessions. In panels B-F, the plasma concentrations were

138 measured throughout the experiment (0-300 min). 0 min refers to morning (pre-meal) levels, after

139 which participants consumed the standard breakfast (shown as an arrow in B). In all the panes, 30

140 min indicates the response after the breakfast. (B) Leptin decreased at month 1 during the diet

141 compared to baseline, but was not significantly different at month 3 compared with month 1.

142 Glucose (C) and Insulin (D) did not show significant differences across sessions. Total ghrelin (E) 
143 and acylated ghrelin (F) levels were lower at month 1 and month 3 compared with baseline. Data

144 are presented as mean \pm SEM. Statistics are derived from linear mixed models (MATLAB

145 function fitlme). ${ }^{*}=\mathrm{p}<0.05 ;{ }^{*}{ }^{*}=\mathrm{p}<0.01$.

147 Activity in cognitive control networks correlated with weight loss

148 We hypothesized that weight loss would be related to activity in regions implicated in cognitive

149 control. In line with this hypothesis, initial weight loss at month 1 was correlated with an increase in

150 BOLD (month 1 versus baseline) during the food minus scenery contrast in regions associated

151 with cognitive control such as dIPFC, IFG, dACC, inferior parietal lobule, and caudate (Fig. 3-

152 A\&B, Table S5). This result is in line with studies that showed that cue-related dlPFC activity

153 correlated with weight loss from dieting (Weygandt et al., 2013) or bariatric surgery (Goldman et

154 al., 2013). Food cue-reactivity in the network of regions related to cognitive control at month 1 (Fig

155 3-A) correlated positively with subsequent weight loss from month 1 to $3(\mathrm{r}=0.60, \mathrm{p}=0.013$, Fig.

$1563-\mathrm{C})$. Moreover, activity reductions in this network at month 3 (i.e. a return towards baseline)

157 correlated with weight regain two years later $(r=-0.64, p=0.014$, Fig. 3-D). These results suggest

158 that engagement of prefrontal areas implicated in dietary self-control is correlated with initial weight

159 loss at months 1 and 3 and weight loss maintenance at two years.

160

161

162

163

164 
A
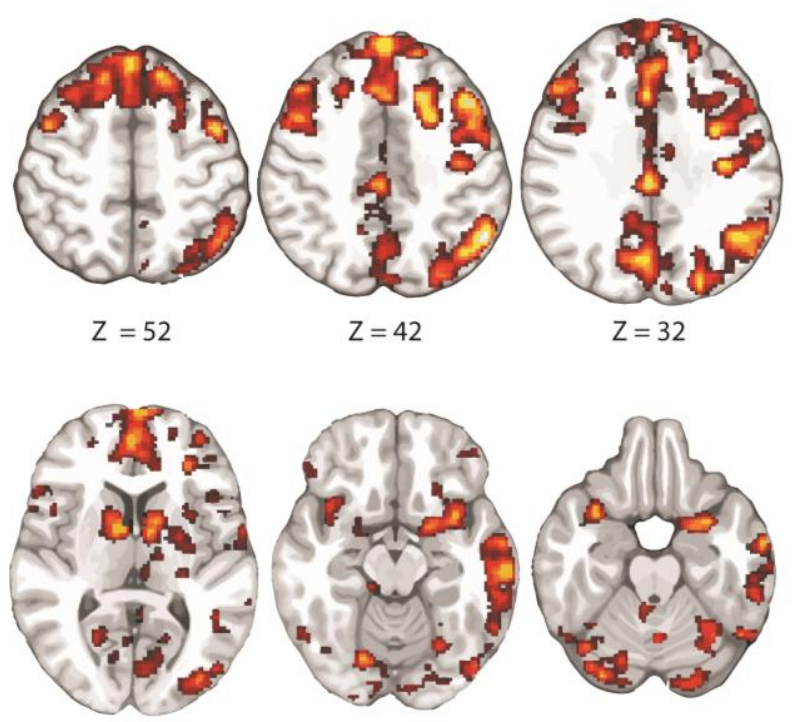

$\mathrm{Z}=8$

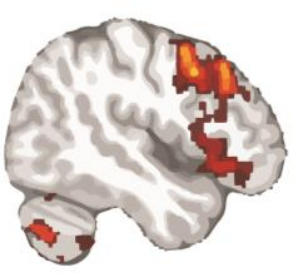

$X=46$

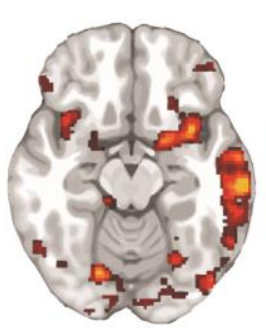

$Z=-15$

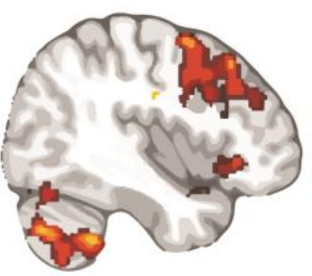

$X=42$

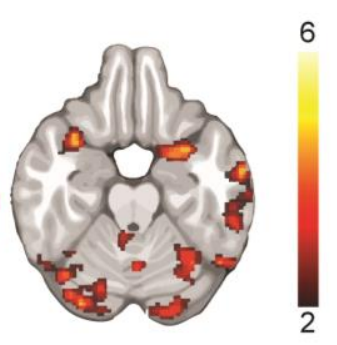

$Z=-22$

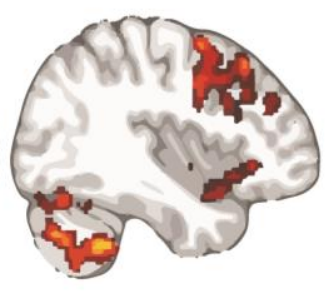

$X=39$
B
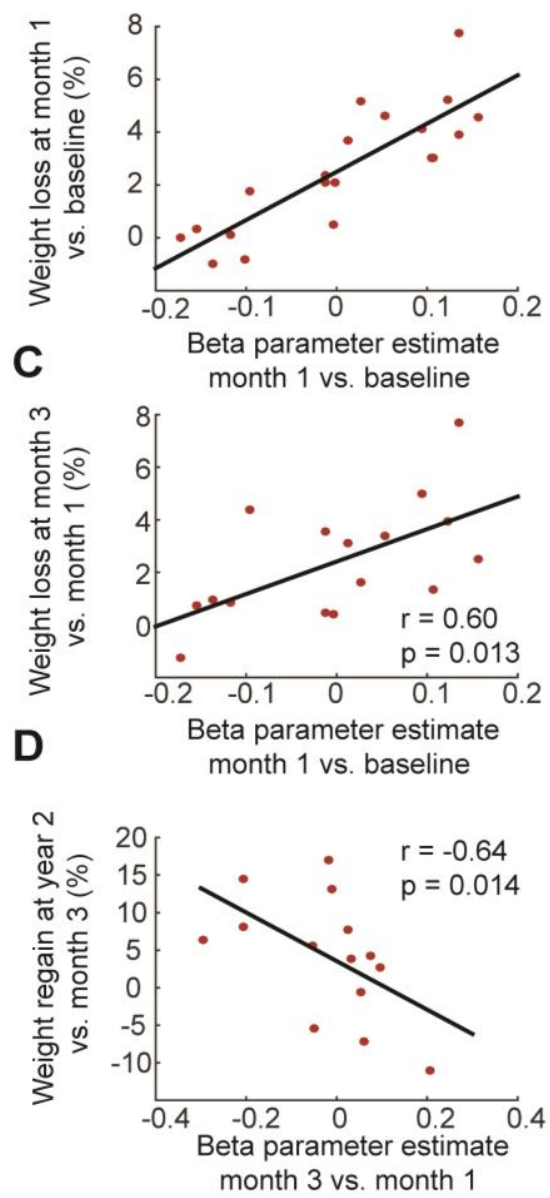

166 Figure 3. Weight loss at month 1 correlated with changes in BOLD in regions associated with

167 cognitive control.

168 (A) Activation to food cues compared to scenery cues at month 1 vs. baseline correlated with

169 weight loss $(\mathrm{p}<0.05$ FWER, $\mathrm{N}=20)$. (B) Mean beta estimate of activation to food minus scenery at

170 month 1 minus baseline derived from the significant cluster (A) versus weight loss between month

1711 and baseline. (C) Mean beta estimate of activation to food minus scenery at month 1 minus

172 baseline derived from the significant cluster (A) versus weight loss from month 3 to month 1 ( $\mathrm{N}=$ 
173 16). (D) Mean beta estimate of activation to food minus scenery at month 3 minus month 1

174 derived from the significant cluster (A) versus weight regain at two years compared with month 3

$175(\mathrm{~N}=14)$. The color scale presents t-statistics derived from 5000 permutations of the data. FWER:

176 Family-Wise Error Rate. X and Z refer to the MNI coordinates in mm.

\section{vmPFC BOLD decreased during calorie restriction}

179 Neuro-computational models of decision-making assume that individuals make choices based on

180 the subjective value assigned to options (Rangel, 2013). As an example, a subjective value signal for

181 food stimuli might be computed by incorporating aspects of palatability and healthiness. Meta-

182 analysis reveals that this stimulus value computation is reflected in the vmPFC (Bartra et al., 2013),

183 and influenced by its connectivity with regions implicated in reward processing, such as the

184 striatum, and cognitive control, such as the dlPFC and IFG (Hare et al., 2009, 2011; Rangel, 2013).

185 We tested two competing predictions regarding the change in vmPFC response to food cues

186 during calorie restriction: (1) it could increase, reflecting greater valuation of food cues due to

187 negative energy balance; (2) it could show a reduction, reflecting successful self-regulation (Hare et

188 al., 2009). Food cue reactivity was reduced in vmPFC at month 1 compared to baseline in a region

189 of interest (ROI) derived from a meta-analysis of subjective value (Bartra et al., 2013) (Fig. 4-A;

190 Table S6A). Activity in vmPFC in the food minus scenery contrast remained lower than baseline at

191 month 3 (Fig. 4-B) in line with continued weight loss. There was no difference in vmPFC activation

192 (food minus scenery) at month 3 compared to month 1. 


\section{Food liking decreased during calorie restriction}

195 Subjective liking ratings for food cues relative to the scenery cues showed a similar trend: there was

196 a significant reduction at month $1\left(\mathrm{~F}(1,61)=283.33, \mathrm{p}=1.34{ }^{*} 10^{-24}\right)$ that persisted at month 3

197 compared with baseline $(\mathrm{F}(1,61)=273.73, \mathrm{p}=0.0012)$. Month 3 liking ratings were not

198 significantly different from month $1(\mathrm{~F}(1,61)=0.23, \mathrm{p}=0.63)$ (Fig. 4-C). These result align with

199 previous observations showing that food cravings subside during voluntary calorie restriction

200 (Martin et al., 2006). Furthermore, liking for food vs. scenery cues correlated with vmPFC food

201 cue reactivity (linear mixed effects model $\mathrm{F}(1,61)=13.24, \mathrm{p}=0.0006)$ across all sessions (Fig. 4-D),

202 supporting a role for this region in value computation. In sum, both fMRI vmPFC signals and

203 liking for food cues were reduced during calorie restriction, consistent with previous studies linking

204 these to self-regulation of appetite (Hare et al., 2009; Wagner et al., 2013).

205

206 vmPFC connectivity changed during calorie restriction

207 The connectivity of the vmPFC (seed region MNI coordinates: $x=-10, y=34, z=-12$, Fig. 4-E),

208 was reduced at month 1 vs baseline with regions associated with visual processing of food stimuli.

209 These regions included the lingual gyrus, the lateral and temporal occipital cortex regions (Fig. 4-E,

210 Table S6B). Focusing on the visual features of valued stimuli has been associated with BOLD

211 response in these regions (van der Laan et al., 2011; Lim et al., 2011), which have been postulated

212 to send the visual information to the vmPFC, where an overall subjective value of the stimulus is

213 computed and utilized for decision making (Lim et al., 2013). Weight loss also correlated with

214 increased vmPFC connectivity to left dIPFC in regions previously associated with cognitive control 
215 (by using a mask generated from the search term "cognitive-control” in the meta-analytical tool

216 Neurosynth) (Fig.4-F, Table S6C).

A
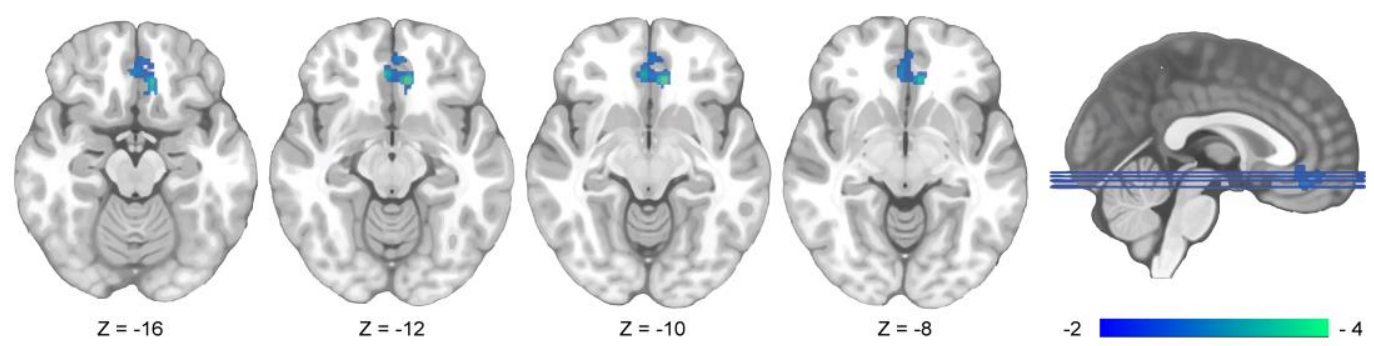

B
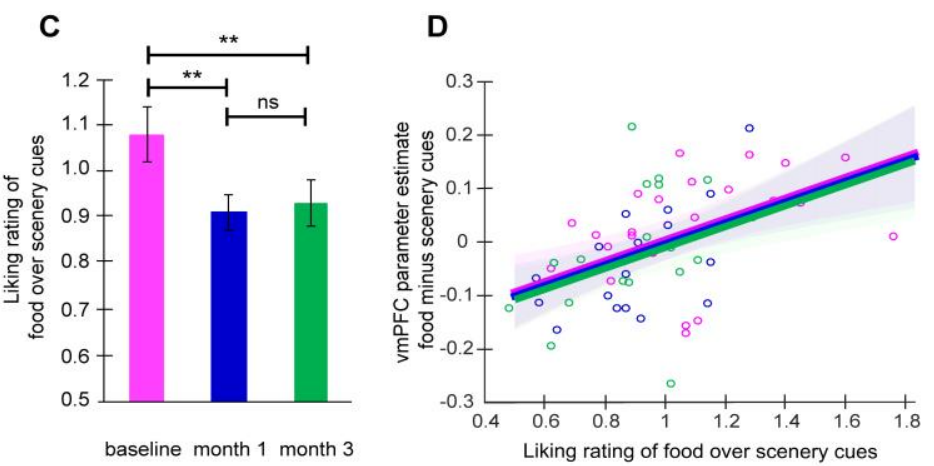

E

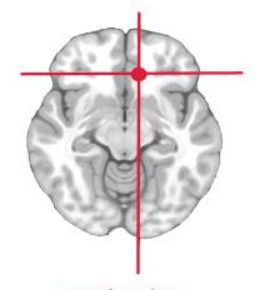

seed region

$\mathbf{F}$

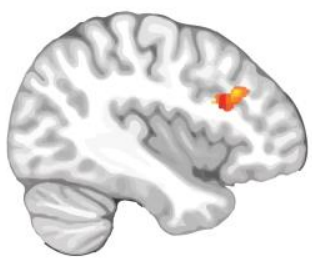

$x=-41$

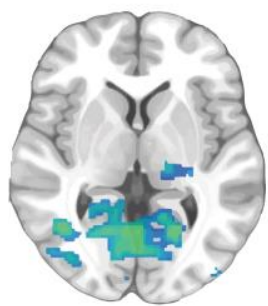

$Z=2$

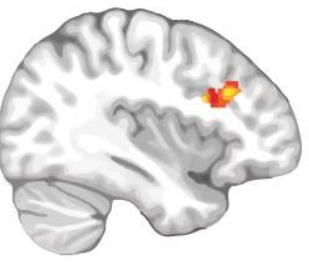

$X=-38$

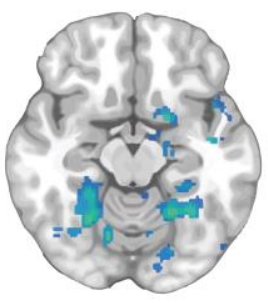

$Z=-15$

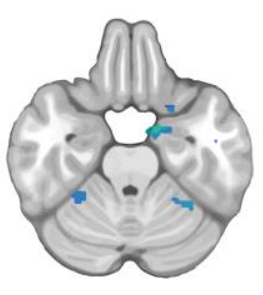

$Z=-25$
G

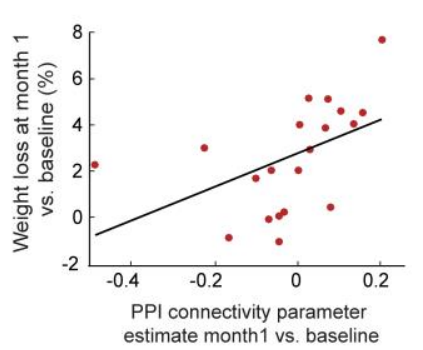




\section{Figure 4. vmPFC activity following calorie restriction}

219 (A) Negative peaks: activation to food cues compared to scenery cues at month 1 of the diet 220 compared to baseline ( $p<0.05$ FWER, SVC, $N=20$ ). (B) Changes in the vmPFC region beta

221 estimate derived from the entire cluster in A. (C) Liking for food cues relative to scenery cues at 222 baseline, month 1 and month 3. (D) Mean vmPFC parameter estimate derived from the entire 223 cluster to food minus scenery cues versus mean liking ratings of food cues relative to scenery cues $224(\mathrm{~F}(1,61)=13.24, \mathrm{p}=0.0006)$. Shaded lines represent 95\% confidence intervals derived from the 225 linear mixed effect model (MATLAB fitlme). (E) PPI analysis with the vmPFC seed revealed that 226 left vmPFC connectivity is reduced with visual areas at month 1 compared with baseline (displayed $227 \mathrm{p}<0.001$, uncorrected, minimum voxel extent $=10 \mathrm{~mm}, \mathrm{p}<0.05$ FWER corrected in Table S6-C).

228 (F) Left vmPFC connectivity to left dIPFC and left IFG at month 1 compared with baseline is 229 positively correlated with weight loss (displayed $\mathrm{p}<0.001$, uncorrected, minimum voxel extent $=10$ $230 \mathrm{~mm}, \mathrm{p}<0.05$ FWER corrected in Table S6-D). (G) The mean beta estimate derived from the 231 significant cluster $(\mathbf{F})$ in month 1 compared with baseline versus weight loss between month 1 and 232 baseline. Data are presented as mean \pm SEM and statistics are derived from linear mixed models.

233 SVC: Small Volume Correction. FWER: Family-wise Error Rate. ns: not significant. ${ }^{*}=$ p<0.01.

$234 \mathrm{X}$ and $\mathrm{Z}$ refer to MNI coordinates in $\mathrm{mm}$. 


\section{IFG activity subsided from month 1 to 3 during calorie restriction}

240 These effects tended to subside from month 1 to 3 , when there was a reduction in activity in right

241 IFG/Frontal Opercular Cortex (Fig. S1-A; Table S6D), and reduced vmPFC connectivity to left

242 dlPFC (Fig. S1-B; Table S7). Participants who showed more reduction in right IFG BOLD at

243 month 3 vs. month 1 showed greater weight-regain at 2-year follow-up $(\mathrm{r}=-0.61, \mathrm{p}=0.02, \mathrm{n}=14$,

244 Fig. S1-B).

246 These results support a model in which calorie restriction entails changes in value computations in

247 vmPFC due to reduced connectivity with visual areas involved in computing attributes of stimuli

248 (Lim et al., 2013) and increased connectivity with prefrontal areas implicated in self-control

249 (Rangel, 2013). These results build on previous work showing that dlPFC activity and its

250 connectivity to vmPFC correlates with self-regulation and reduced food consumption (Hare et al.,

251 2009; Lopez et al., 2014). In this study, changes in brain activity in these regions predicted

252 subsequent real-life outcomes and correlated with the magnitude of weight loss. However, we

253 observed that both the activity in regions associated with cognitive control as well as their

254 connectivity to vmPFC were reduced at month 3 compared with month 1 suggesting that BOLD

255 changes in cognitive control networks may not be sustained, which appears to correlate with

256 subsequent weight regain (Fig. 3-D).

258 Leptin decreased, and ghrelin increased during calorie restriction

259 Leptin and ghrelin both showed adaptations to weight loss. Plasma leptin showed significant effects

260 for both session $\left(\mathrm{F}(1,188)=81.91, \mathrm{p}=1.79^{*} 10^{-16}\right)$ and sampling time $(\mathrm{F}(1,188)=7.29, \mathrm{p}=$ 

significantly different from baseline $(\mathrm{F}(1,187)=2.30, \mathrm{p}=0.13)$.

$272\left(\mathrm{~F}(1,376)=46.5, \mathrm{p}=4.54{ }^{*} 10^{-11}\right)$. During the scan ghrelin increased further $(\mathrm{F}(1,376)=41.81, \mathrm{p}=$ $\left.2733.11 * 10^{-10}\right)$, to a level not different from time $\left.0 \mathrm{~F}(1,376)=1.6, \mathrm{p}=0.21\right)$.

275 Plasma acyl-ghrelin (Fig.2-F) showed a main effect of increase at month $1(\mathrm{~F}(1,379)=5.00, \mathrm{p}=$ 276 0.026), remaining elevated at month 3 relative to baseline $\mathbf{F}(1,379)=4.27, \mathrm{p}=0.039)$. They were 277 not different between months 1 and $3(\mathrm{~F}(1,379)=0.025, \mathrm{p}=0.87)$. As expected, levels decreased 278 markedly by 60 minutes following breakfast $\left(\mathrm{F}(1,376)=22.63, \mathrm{p}=2.81 * 10^{-6}\right)$ then rose 279 progressively thereafter. Levels at the end of scan $(300 \mathrm{~min})$ were not different than at time $0(\mathrm{~F}$ $280(1,376)=2.2, \mathrm{p}=0.14)$, but increased during the scan $(180 \mathrm{vs} .300 \mathrm{~min})(\mathrm{F}(1,376)=20.39, \mathrm{p}=$ $\left.2818.46^{*} 10^{-6}\right)$. 
283 The magnitude of month 1 weight loss was correlated with pre-meal log leptin and ghrelin levels. It

284 was negatively correlated with pre-meal $\log$ leptin $\left(\mathrm{r}=-0.78, \mathrm{p}=1.56^{*} 10^{-5}\right.$, Fig. 5-A), and positively

285 with pre-meal total ghrelin levels at month $1\left(\mathrm{r}=0.67, \mathrm{p}=7.41{ }^{*} 10^{-4}\right.$, Fig. 5-B) but not with acyl-

286 ghrelin levels $(r=0.20, \mathrm{p}=0.38)$. The effects remained at month 3 when weight loss compared

287 with baseline correlated inversely with pre-meal leptin levels $\left(\mathrm{r}=-0.86, \mathrm{p}=5.39{ }^{*} 10^{-6}\right)$ and

288 positively with pre-meal acyl ghrelin $(\mathrm{r}=0.47, \mathrm{p}=0.048)$ and total ghrelin levels $(\mathrm{r}=0.56, \mathrm{p}=$

$2890.016)$.

290

291 No significant effects of session on glucose and insulin levels were detected (Fig. 2 C-D). In

292 addition, insulin sensitivity as assessed by homeostatic model assessment (HOMA) was not

293 significantly different across sessions $(\mathrm{F}(1,61)=1.37, \mathrm{p}=0.25$, Table $\mathrm{S} 2)$. These results are

294 consistent with insulin resistance playing a minor role in the main outcome variables in this study.

295

296 Leptin is produced by adipocytes. Its plasma levels fall quickly during negative energy balance, and

297 more gradually with diminished fat mass (Friedman \& Mantzoros, 2015). The orexigenic hormone

298 ghrelin is increased in response to energy deficit (Borer et al., 2009; Müller et al., 2015). In the

299 current study, leptin levels decreased while ghrelin levels increased at month 1, consistent with

300 previous weight loss studies (Wing et al., 1996). Despite continued weight loss, ghrelin and leptin

301 levels did not change further at month 3, as described previously (Crujeiras et al., 2010; Sumithran

302 et al., 2011) These results suggest that, on average, our participants were in negative energy balance

303 at month 1 and month 3 compared with baseline. 


\section{Ghrelin and leptin changes correlated with BOLD in reward regions}

306 We tested the hypothesis that weight loss-induced changes in ghrelin and leptin would increase

307 brain activation of reward related areas while viewing food cues, and that this would promote over308 eating and diet failure. Previous studies found that leptin administration reduced food cue

309 reactivity in leptin deficient patients (Aotani et al., 2012; Farooqi et al., 2007); while ghrelin

310 administration increased food cue reactivity (Malik et al., 2008) in regions associated with food

311 value and motivation.

312 Consistent with previous studies (Goldstone et al., 2014; Kroemer et al., 2013; Malik et al., 2008),

313 increased pre-meal ghrelin levels correlated with increased activity at month 1 in mPFC, caudate

314 and visual cortex, all areas associated with appetitive processing (Fig. 5C,E-F; Table S8). Weight

315 loss-induced reductions in pre-meal leptin level also correlated with increased activity in mPFC and

316 visual cortex (Fig. 5C-D, Table S9), suggesting that metabolic adaptations at month 1 of

317 contributed to increased food cue reactivity at month 1 compared with baseline.

\section{Ghrelin and leptin changes did not oppose continued weight loss}

320 The next question we addressed was whether negative energy balance signals could counteract

321 participants' efforts to continue losing weight by increasing value-related food cue reactivity and

322 food intake. Contrary to our hypothesis, leptin reductions at month 1 vs. baseline correlated with

323 further weight loss at month 3 vs. month $1(\mathrm{r}=-0.69, \mathrm{p}=0032$, Fig. 5-G) and a lower weight at two

324 years compared with baseline $(r=0.57, \mathrm{p}=0.02$, Fig. 5-H). Increases in ghrelin levels did not

325 significantly relate to weight change at 3 month $(r=0.43, \mathrm{p}=0.099)$, or at two years compared with

326 baseline $(r=-0.34, p=0.20)$, however the direction of the effect was not consistent with ghrelin 
327 causing an increase in weight. These results suggest that leptin reductions and ghrelin increases did

328 not oppose continued weight loss, even though they increased reward related food cue-reactivity.

329 Indeed, leptin reductions predicted greater subsequent weight loss. This is likely because leptin

330 reductions were greater in individuals with better appetite control, who lost more weight. 
A

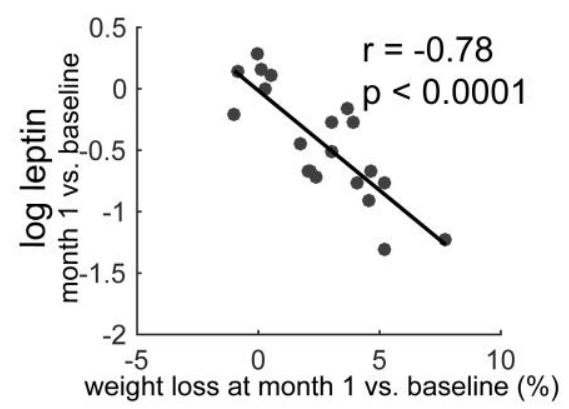

C

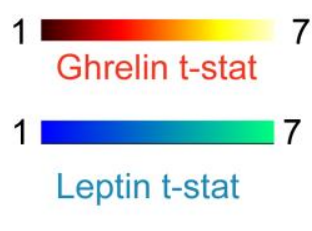

Leptin \& Ghrelin

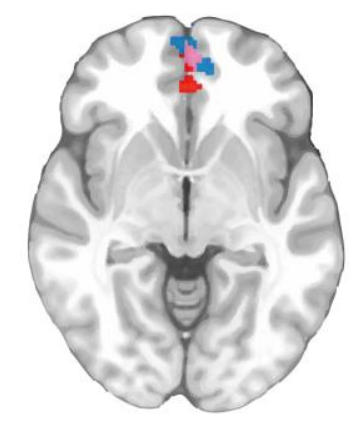

$$
Z=-5
$$

E

1 Ghrelin t-stat

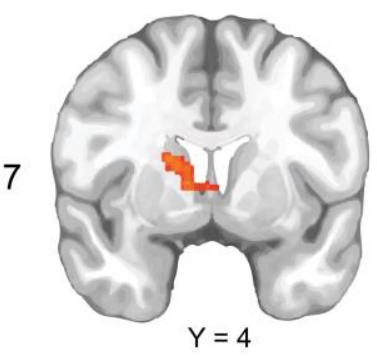

G

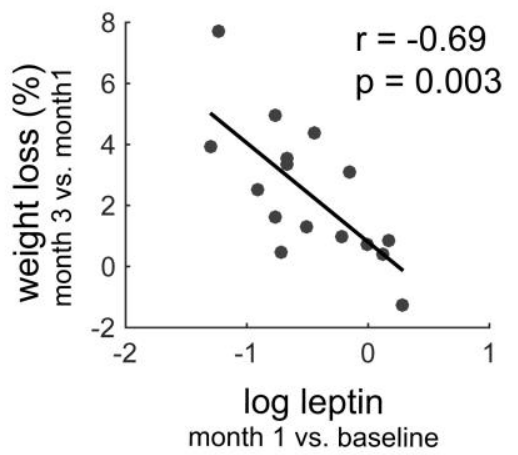

B
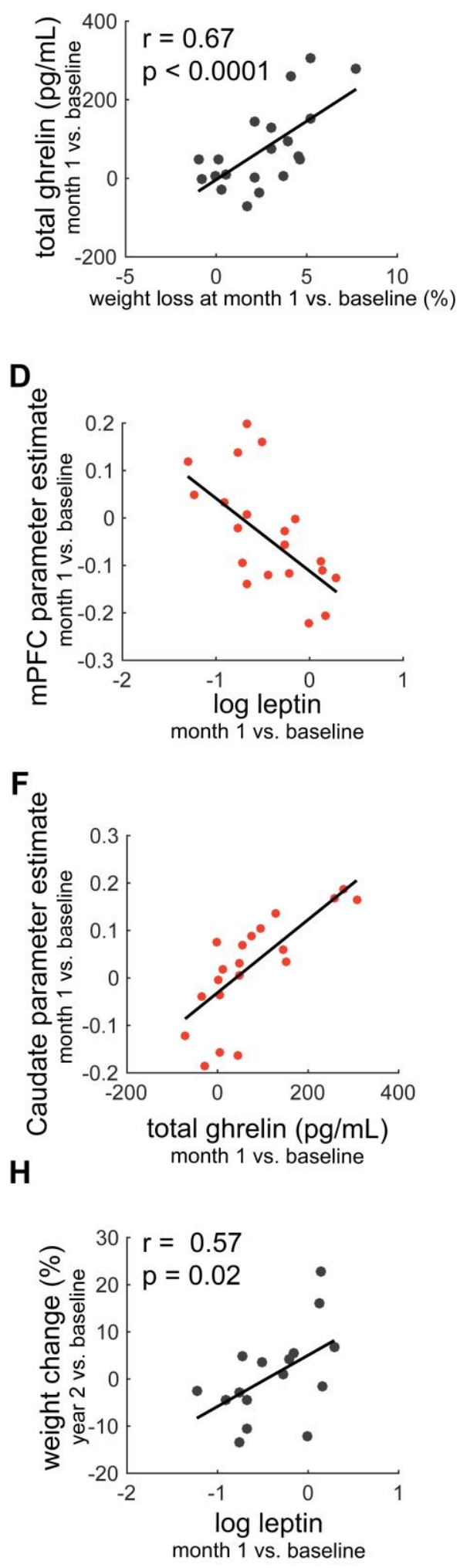


\section{Figure.5 Correlations with leptin and ghrelin levels}

333 (A) Weight loss at month 1 vs. baseline versus pre-meal plasma leptin and (B) total ghrelin at

334 month 1 vs. baseline. (C) Correlation of food minus scenery activation at month 1 vs. baseline and

335 the increase in pre-meal ghrelin levels (orange); and the reduction in pre-meal log leptin levels

336 (blue) at month 1 vs. baseline. Pink regions display areas significant in both analyses (displayed p

$337<0.05$ FWER, Table S6A). (D) Depiction of the correlation from panel C. The mean beta

338 parameter estimate is derived from the orange cluster in panel $\mathrm{C}$ at month 1 vs. baseline. (E)

339 Correlation of food minus scenery activation at month 1 vs. baseline and the increase in pre-meal

340 ghrelin levels (orange); (displayed p <0.05 FWER, Table S6A). (F) Depiction of the correlation

341 from panel E. The mean beta parameter estimate was derived from the orange cluster in panel $\mathrm{E}$

342 (G) Pre-meal $\log$ leptin levels at month 1 vs. baseline correlated inversely with future weight loss at

343 month 3 vs. month 1 and $(\mathrm{H})$ negatively with weight at two years compared with baseline. The

344 color scales represent t-statistics derived from 5000 permutations of the data. FWER: Family-Wise

345 Error Rate. Y and Z refer to MNI coordinates in mm.

347 In this study, leptin and ghrelin adaptations to weight loss did not explain why diets are 348 unsustainable in the long term. Our results suggest that the reduction of leptin at month 1 are a 349 marker of successful weight loss, correlating with future weight loss and/or maintenance. Indeed, in 350 the Look Ahead study, weight loss at month 1 predicted reduced weight up to 8 years later (Unick 351 et al., 2015). Our results are not consistent with the prevalent view that reduced leptin levels during 352 energy restriction promote increased food intake and obesity, a theory that has been recently 353 questioned (Flier and Maratos-Flier, 2017). A recent meta-analysis of reduced leptin levels as a 
354 predictor for weight regain was also inconclusive (Strohacker et al., 2014) and leptin

355 pharmacotherapy in the treatment of obesity has produced disappointing results (Bray, 2014;

356 Korner et al., 2013). All together, these results do not support pharmacologically increasing leptin

357 signaling as an adjunct target for treatment of obesity in conjunction with calorie restriction.

358 However, more research is needed to test the role of leptin for the treatment of obesity in

359 normoleptinemic patients (Friedman and Mantzoros, 2015; Rosenbaum and Leibel, 2014).

361 Conclusions

362 Weight loss is highly variable among individuals embarking on weight-reduction programs and

363 likely depends on interactions between peripheral and central mechanisms of appetite control.

364 While changes in short- and long-term energy signals due to weight loss can affect food intake to

365 maintain a stable weight (Ryan, Woods, \& Seeley, 2012), in humans food intake is also under the

366 influence of cognitive goals and self-regulation. It is important to note that cognitive control

367 changes were not measured here but inferred from the activation of lateral prefrontal areas while

368 viewing food items. Nonetheless, our principal findings are that changes in neural correlates of

369 cognitive control from baseline to month 1 were associated with the magnitude of weight loss at

370 months 1 and 3, while reduced activity from months 1 to 3 in these regions correlated with

371 subsequent weight regain. In addition, increased ghrelin and reduced leptin levels were observed

372 during calorie restriction, but these responses, while increasing food-cue reactivity in reward related

373 brain regions, did not counteract further weight-loss. Thus, we failed to show that hormonal

374 adaptations to negative energy balance play a significant negative role in weight loss success and its 
375 maintenance. Therefore, it is possible that strategies that target the central brain networks involved

376 in cognitive control might be more effective as weight loss strategies.

378 Limitations

379 Although our small sample size is common for within-design neuroimaging studies, it limits our 380 statistical power and the generalizability of our findings. In addition, for some participants we 381 utilized self-reported weight at 2 years, which might have biased our results. Second, we only tested 382 total ghrelin, acyl-ghrelin, leptin, glucose and insulin levels. Therefore, we cannot generalize our 383 results to other metabolic signaling molecules such as glucagon-like peptide (GLP-1). Third, the 384 communication between the metabolic hormones and the brain might have changed as a function 385 of weight status (Ravussin et al, 2014); e.g., leptin resistance might have been present in our 386 sample. We did not test for hormonal resistance, and therefore cannot determine how much 387 changes in peripheral signaling affected brain responses at different time points. Nonetheless, 388 leptin and ghrelin did have the expected effects on cue-reactivity in appetitive brain regions. 389 Another limitation is the lack of a control group. Further studies are justified, and could include 390 larger cohorts, a control group, monitoring of daily food intake and activity, indirect calorimetry,

391 more precise estimation of insulin sensitivity, and additional known modulators of food intake and 392 activity. 


\section{Acknowledgments}

395 We thank Dr. Isabelle Garcia-Garcia and Dr. Andreanne Michaud for critically reading the 396 manuscript. AD is supported by Canadian Institutes of Health Research (CIHR) Foundation

397 Scheme. SN is supported by the Frederick Banting and Charles Best Canada Graduate 398 Scholarship (CIHR). This projected was funded by CIHR Operating Grant number 219271.

\section{Author Contributions}

401 Conceptualization, S.N, W.H., E.B.M and A.D.; Methodology, S.N., W.H., K.L, M.L., E.B.M.,

402 M.D. and Y.Z.; Software, S.N., M.D. and K.L.; Investigation, S.N., W.H, M.Z., S.G.S., M.L.,

403 S.C.S, M.L.; Writing - Original Draft, S.N. and A.D.; Writing - Review \& Editing, S.N., M.D.,

404 E.B.M. and A.D.; Funding Acquisition, E.M. and A.D.; Resources, E.B.M., M.L., S.S. and A.D.;

405 Supervision, E.B.M. and A.D.

406

407 Declarations of Interest

408 Maurice Larocque is the founder and owner of Clinique Motivation Minceur. He recruited the

409 participants, but did not fund the study. The other authors declare no conflict of interest.

\section{$411 \quad$ References}

412 Anastasiou, C.A., Karfopoulou, E., and Yannakoulia, M. (2015). Weight regaining: From

413 statistics and behaviors to physiology and metabolism. Metabolism. 64, 1395-1407.

414 Andersson, J.L.R., Jenkinson, M., Smith, S., and Andersson, J. (2007). Non-linear optimisation 
FMRIB Technial Report TR07JA1.

416 Aotani, D., Ebihara, K., Sawamoto, N., Kusakabe, T., Aizawa-Abe, M., Kataoka, S., Sakai, T.,

417 Iogawa, H., Ebihara, C., Fujikura, J., et al. (2012). Functional magnetic resonance imaging

418 analysis of food-related brain activity in patients with lipodystrophy undergoing leptin

419 replacement therapy. J. Clin. Endocrinol. Metab. 97, 3663-3671.

420 Bartra, O., McGuire, J.T., and Kable, J.W. (2013). The valuation system: a coordinate-based

421 meta-analysis of BOLD fMRI experiments examining neural correlates of subjective value.

422 Neuroimage 76, 412-427.

423 Batterink, L., Yokum, S., and Stice, E. (2010). Body mass correlates inversely with inhibitory

424 control in response to food among adolescent girls: an fMRI study. Neuroimage 52, 1696-1703.

425 Beck A.T., B.R.W. (1972). Screening depressed patients in family practice: A rapid technique.

426 Postgrad. Med. 52, 81-85.

427 Beckmann, C.F., Jenkinson, M., and Smith, S.M. (2003). General multilevel linear modeling for 428 group analysis in FMRI. Neuroimage 20, 1052-1063.

429 Berthoud, H.-R., Zheng, H., and Shin, A.C. (2012). Food reward in the obese and after weight

430 loss induced by calorie restriction and bariatric surgery. Ann. N. Y. Acad. Sci. 1264, 36-48.

431 Borer, K.T., Wuorinen, E., Ku, K., and Burant, C. (2009). Appetite responds to changes in meal 432 content, whereas ghrelin, leptin, and insulin track changes in energy availability. J. Clin.

433 Endocrinol. Metab. 94, 2290-2298.

434 Bray, G.A. (2014). Medical treatment of obesity: The past, the present and the future. Best Pract. 435 Res. Clin. Gastroenterol. 28, 665-684.

436 Carver, C.S., and White, T.L. (1994). Behavioral inhibition, behavioral activation, and affective 
437 responses to impending reward and punishment: The BIS/BAS Scales. J. Pers. Soc. Psychol. 67,

$438 \quad 319-333$.

439 Cohen, S., Kamarck, T., and Mermelstein, R. (1983). A global measure of perceived stress. J.

$440 \quad$ Health Soc. Behav. 24, 385-396.

441 Crujeiras, A.B., Goyenechea, E., Abete, I., Lage, M., Carreira, M.C., Martínez, J.A., and

442 Casanueva, F.F. (2010). Weight Regain after a Diet-Induced Loss Is Predicted by Higher

443 Baseline Leptin and Lower Ghrelin Plasma Levels. J. Clin. Endocrinol. Metab. 95, 5037-5044.

444 Dagher, A. (2012). Functional brain imaging of appetite. Trends Endocrinol. Metab. 23, 250-

445260.

446 Eskildsen, S.F., Coupé, P., Fonov, V., Manjón, J. V., Leung, K.K., Guizard, N., Wassef, S.N.,

447 Østergaard, L.R., Collins, D.L., and Alzheimer's Disease Neuroimaging Initiative (2012).

448 BEaST: Brain extraction based on nonlocal segmentation technique. Neuroimage 59, 2362-

4492373.

450 Farooqi, I.S., Bullmore, E., Keogh, J., Gillard, J., O’Rahilly, S., and Fletcher, P.C. (2007). Leptin

451 regulates striatal regions and human eating behavior. Science 317, 1355.

452 Flier, J.S., and Maratos-Flier, E. (2017). Leptin’s Physiologic Role: Does the Emperor of Energy

453 Balance Have No Clothes? Cell Metab. 26, 24-26.

454 Friedman, J.M., and Mantzoros, C.S. (2015). 20 years of leptin: From the discovery of the leptin 455 gene to leptin in our therapeutic armamentarium. Metabolism. 64, 1-4.

456 Gettens, K.M., and Gorin, A.A. (2017). Executive function in weight loss and weight loss

457 maintenance: a conceptual review and novel neuropsychological model of weight control. J.

458 Behav. Med. 1-15. 
Goldstone, A.P., Prechtl, C.G., Scholtz, S., Miras, A.D., Chhina, N., Durighel, G., Deliran, S.S.,

460 Beckmann, C., Ghatei, M.A., Ashby, D.R., et al. (2014). Ghrelin mimics fasting to enhance

461 human hedonic, orbitofrontal cortex, and hippocampal responses to food. Am. J. Clin. Nutr. 99,

462 1319-1330.

463 Grosshans, M., Vollmert, C., Vollstädt-Klein, S., Tost, H., Leber, S., Bach, P., Bühler, M., von

464 der Goltz, C., Mutschler, J., Loeber, S., et al. (2012). Association of leptin with food cue-induced

465 activation in human reward pathways. Arch. Gen. Psychiatry 69, 529-537.

466 Gudzune, K.A., Doshi, R.S., Mehta, A.K., Chaudhry, Z.W., Jacobs, D.K., Vakil, R.M., Lee, C.J.,

467 Bleich, S.N., and Clark, J.M. (2015). Efficacy of Commercial Weight-Loss Programs: An

468 Updated Systematic Review. Ann. Intern. Med. 162, 501-512.

469 Hare, T.A., Camerer, C.F., and Rangel, A. (2009). Self-Control in Decision-Making Involves

470 Modulation of the vmPFC Valuation System. 324.

471 Hare, T.A., Malmaud, J., and Rangel, A. (2011). Focusing attention on the health aspects of

472 foods changes value signals in vmPFC and improves dietary choice. J. Neurosci. 31, 11077-

47311087.

474 Hollmann, M., Hellrung, L., Pleger, B., Schlögl, H., Kabisch, S., Stumvoll, M., Villringer, A., 475 and Horstmann, A. (2012). Neural correlates of the volitional regulation of the desire for food.

476 Int. J. Obes. (Lond). 36, 648-655.

477 Hosoda, H., and Kangawa, K. (2012). Standard sample collections for blood ghrelin

478 measurements. Methods Enzymol. 514, 113-126.

479 Jenkinson, M., Bannister, P., Brady, M., and Smith, S. (2002). Improved optimization for the 480 robust and accurate linear registration and motion correction of brain images. Neuroimage 17, 
$825-841$.

482 Jenkinson, M., Beckmann, C.F., Behrens, T.E.J., Woolrich, M.W., and Smith, S.M. (2012). FSL.

483 Neuroimage 62, 782-790.

484 Korner, J., Conroy, R., Febres, G., McMahon, D.J., Conwell, I., Karmally, W., and Aronne, L.J.

485 (2013). Randomized double-blind placebo-controlled study of leptin administration after gastric

486 bypass. Obesity (Silver Spring). 21, 951-956.

487 Kroemer, N.B., Krebs, L., Kobiella, A., Grimm, O., Pilhatsch, M., Bidlingmaier, M.,

488 Zimmermann, U.S., and Smolka, M.N. (2013). Fasting levels of ghrelin covary with the brain

489 response to food pictures. Addict. Biol. 18, 855-862.

490 van der Laan, L.N., de Ridder, D.T.D., Viergever, M. a., and Smeets, P. a M. (2011). The first

491 taste is always with the eyes: A meta-analysis on the neural correlates of processing visual food

492 cues. Neuroimage 55, 296-303.

493 Lim, S.-L., O’Doherty, J.P., and Rangel, A. (2011). The decision value computations in the

494 vmPFC and striatum use a relative value code that is guided by visual attention. J. Neurosci. 31,

$495 \quad 13214-13223$.

496 Lim, S.-L., O’Doherty, J.P., and Rangel, A. (2013). Stimulus value signals in ventromedial PFC

497 reflect the integration of attribute value signals computed in fusiform gyrus and posterior

498 superior temporal gyrus. J. Neurosci. 33, 8729-8741.

499 Lopez, R.B., Hofmann, W., Wagner, D.D., Kelley, W.M., and Heatherton, T.F. (2014). Neural

500 Predictors of Giving in to Temptation in Daily Life. Psychol. Sci. 25, 1337-1344.

501 Lopez, R.B., Chen, P.-H.A., Huckins, J.F., Hofmann, W., Kelley, W.M., and Heatherton, T.F.

502 (2017). A balance of activity in brain control and reward systems predicts self-regulatory 
outcomes. Soc. Cogn. Affect. Neurosci. 12, 832-838.

504 Lowe, M.R., Butryn, M.L., Didie, E.R., Annunziato, R.A., Thomas, J.G., Crerand, C.E., Ochner,

505 C.N., Coletta, M.C., Bellace, D., Wallaert, M., et al. (2009). The Power of Food Scale. A new

506 measure of the psychological influence of the food environment. Appetite 53, 114-118.

507 Malik, S., McGlone, F., Bedrossian, D., and Dagher, A. (2008). Ghrelin Modulates Brain

508 Activity in Areas that Control Appetitive Behavior. Cell Metab. 7, 400-409.

509 Martin, C.K., O’Neil, P.M., and Pawlow, L. (2006). Changes in Food Cravings during Low-

510 Calorie and Very-Low-Calorie Diets*. Obesity 14, 115-121.

511 Michaud, A., Vainik, U., Garcia-Garcia, I., and Dagher, A. (2017). Overlapping Neural

512 Endophenotypes in Addiction and Obesity. Front. Endocrinol. (Lausanne). 8, 127.

513 Müller, T.D., Nogueiras, R., Andermann, M.L., Andrews, Z.B., Anker, S.D., Argente, J.,

514 Batterham, R.L., Benoit, S.C., Bowers, C.Y., Broglio, F., et al. (2015). Ghrelin. Mol. Metab. 4, 515 437-460.

516 Murdaugh, D.L., Cox, J.E., Cook, E.W., and Weller, R.E. (2012). fMRI reactivity to high-calorie

517 food pictures predicts short- and long-term outcome in a weight-loss program. Neuroimage 59,

518 2709-2721.

519 Neseliler, S., Han, J., and Dagher, A. (2017). The Use of Functional Magnetic Resonance

520 Imaging in the Study of Appetite and Obesity. Appet. Food Intake 2, 117-134.

521 O’Reilly, J.X., Woolrich, M.W., Behrens, T.E.J., Smith, S.M., and Johansen-Berg, H. (2012).

522 Tools of the trade: psychophysiological interactions and functional connectivity. Soc. Cogn.

523 Affect. Neurosci. 7, 604-609.

524 Ochner, C.N., Barrios, D.M., Lee, C.D., and Pi-Sunyer, F.X. (2013). Biological mechanisms that 
525 promote weight regain following weight loss in obese humans. Physiol. Behav. 120, 106-113.

526 Rangel, A. (2013). Regulation of dietary choice by the decision-making circuitry. Nat. Neurosci.

$527 \quad 16,1717-1724$.

528 Ravussin, Y., LeDuc, C.A., Watanabe, K., Mueller, B.R., Skowronski, A., Rosenbaum, M., and

529 Leibel, R.L. (2014). Effects of chronic leptin infusion on subsequent body weight and

530 composition in mice: Can body weight set point be reset? Mol. Metab. 3, 432-440.

531 Rosenbaum, M., and Leibel, R.L. (2014). 20 years of leptin: role of leptin in energy homeostasis

532 in humans. J. Endocrinol. 223, T83-96.

533 Rosenbaum, M., Sy, M., Pavlovich, K., Leibel, R.L., and Hirsch, J. (2008). Leptin reverses

534 weight loss-induced changes in regional neural activity responses to visual food stimuli. J. Clin.

535 Invest. 118, 2583-2591.

536 Smith, S.M., and Nichols, T.E. (2009). Threshold-free cluster enhancement: addressing problems

537 of smoothing, threshold dependence and localisation in cluster inference. Neuroimage 44, 83-98.

538 Stoeckel, L.E., Birch, L.L., Heatherton, T., Mann, T., Hunter, C., Czajkowski, S., Onken, L.,

539 Berger, P.K., and Savage, C.R. (2017). Psychological and Neural Contributions to Appetite Self-

$540 \quad$ Regulation. Obesity 25, 17-25.

541 van Strien, T., Frijters, J.E.R., Bergers, G.P.A., and Defares, P.B. (1986). The Dutch Eating

542 Behavior Questionnaire (DEBQ) for assessment of restrained, emotional, and external eating

543 behavior. Int. J. Eat. Disord. 5, 295-315.

544 Strohacker, K., McCaffery, J.M., MacLean, P.S., and Wing, R.R. (2014). Adaptations of leptin,

545 ghrelin or insulin during weight loss as predictors of weight regain: a review of current literature.

546 Int. J. Obes. (Lond). 38, 388-396. 
547 Sumithran, P., Prendergast, L.A., Delbridge, E., Purcell, K., Shulkes, A., Kriketos, A., and

548 Proietto, J. (2011). Long-Term Persistence of Hormonal Adaptations to Weight Loss. N. Engl. J.

549 Med. 365, 1597-1604.

550 Torrubia, R., Ávila, C., Moltó, J., and Caseras, X. (2001). The Sensitivity to Punishment and

551 Sensitivity to Reward Questionnaire (SPSRQ) as a measure of Gray's anxiety and impulsivity

552 dimensions. Pers. Individ. Dif. 31, 837-862.

553 Unick, J.L., Neiberg, R.H., Hogan, P.E., Cheskin, L.J., Dutton, G.R., Jeffery, R., Nelson, J.A.,

554 Pi-Sunyer, X., West, D.S., and Wing, R.R. (2015). Weight change in the first 2 months of a

555 lifestyle intervention predicts weight changes 8 years later. Obesity (Silver Spring). 23, 1353-

5561356.

557 Wagner, D.D., Altman, M., Boswell, R.G., Kelley, W.M., and Heatherton, T.F. (2013). Self-

558 regulatory depletion enhances neural responses to rewards and impairs top-down control.

559 Psychol. Sci. 24, 2262-2271.

560 Weygandt, M., Mai, K., Dommes, E., Leupelt, V., Hackmack, K., Kahnt, T., Rothemund, Y.,

561 Spranger, J., and Haynes, J.-D. (2013). The role of neural impulse control mechanisms for

562 dietary success in obesity. Neuroimage 83, 669-678.

563 Wing, R., Sinha, M., Considine, R., Lang, W., and Caro, J. (1996). Relationship Between Weight

564 Loss Maintenance and Changes in Serum Leptin Levels. Horm. Metab. Res. 28, 698-703.

565 Woolrich, M.W., Behrens, T.E.J., Beckmann, C.F., Jenkinson, M., and Smith, S.M. (2004).

566 Multilevel linear modelling for FMRI group analysis using Bayesian inference. Neuroimage 21,

$567 \quad 1732-1747$.

568 Yarkoni, T., Poldrack, R.A., Nichols, T.E., Van Essen, D.C., and Wager, T.D. (2011). Large- 
569 scale automated synthesis of human functional neuroimaging data. Nat. Methods 8, 665-670.

570

571

572

573

574

575

576

577

578

579

580

581

582

583

584

585

586

587 
Materials and Methods

Participants

59129 right-handed participants $[1$ male, $\mathrm{BMI}=30.94(\mathrm{SD}= \pm 3.76)$; age $=37.28(\mathrm{SD}= \pm 7.99)]$ were

592 recruited in a private weight loss clinic (“Clinique Motivation Minceur”, Montréal QC Canada)

593 before they started a prescribed weight-loss regimen. After the protocol was explained, those

594 accepting to participate signed the consent form, underwent medical history, physical examinations

595 and blood testing to look for comorbid conditions. Inclusion criteria were: healthy apart from

596 obesity, and stable weight at least for three months. Exclusion criteria were diabetes, uncontrolled

597 hypertension, currently smoking, substance abuse and current use of a central nervous system

598 active medication, renal disease, non-dermatologic cancer in the previous 5 years and current or

599 history of neurological, eating or psychiatric disorders. Candidates who could not undergo MRI

600 due to claustrophobia, pregnancy, implanted metal, or BMI $>40 \mathrm{~kg} / \mathrm{m}^{2}$ were also excluded $(\mathrm{n}=1)$.

601 Each participant received an individualized non-ketogenic calorie-restricted diet program from the

602 weight loss clinic. The prescribed diets contained $1100-1400 \mathrm{kcal} / \mathrm{day}$, with $40 \%$ carbohydrate, $30 \%$

603 fat and $30 \%$ protein. Oral calcium and multivitamins were recommended, as was $30-45$ min of

604 brisk walking per day. Weekly visits included motivational counselling. The study was approved by

605 the Montreal Neurological Institute Research Ethics Board. Volunteers received compensation for

606 participation in the form of free nutritional supplements and reimbursement for travel.

607

608 Data from one participant was not included due the presence of uncorrectable fMRI brain artifacts

$609(\mathrm{n}=1)$. Lack of button response to more than $25 \%$ of the stimulus ratings deemed a run 
610 incomplete as it indicated that the participant was not paying attention to the task. Any session with

$61150 \%$ incomplete runs (3 out of 6 runs) was excluded. Three participants were inattentive during

612 the fMRI scan during at all 3 sessions $(n=3)$. In addition, four did not successfully complete the

613 fMRI at session 2 (leaving $n=20$ ) and four (others) at session 3 (leaving $n=20$ ). This reduced the

614 sample size for comparing baseline to month 1 to 20 participants, and the comparison of month 1

615 to month 3 to 16 participants. Participants were contacted at two years following the experiment.

616 Of 19 respondents, weights were reported by 10, and measured in 9. Of these 19 respondents, 14

617 people completed all three sessions of the fMRI successfully. Weight at two years is calculated as

618 the percent change of weight from baseline to two years $(n=19)$; and weight regain as the percent

619 change of weight from month 3 to two years $(n=14)$.

\section{Experimental Design}

622 Participants underwent fMRI on three occasions: first immediately prior to initiation of the diet

623 program, then at month 1 and month 3 during calorie restriction. Women were scanned during

624 the luteal phase of their menstrual cycle. The scanning sessions started between 12:00 and 1:30

625 PM and kept the same schedule for the three sessions. On the scan days, participants presented

626 themselves to the lab in the morning, having fasted from midnight the night before. All participants

627 received the same standardized breakfast. This included $3 / 4$ cup of $2 \%$ milk, 1 slice of brown toast,

$62810 \mathrm{~mL}$ of peanut butter, one medium-sized hard -boiled egg, one small apple, a protein bar and

$629150 \mathrm{~mL}$ of black coffee or tea without sugar. It contained $550 \mathrm{kcal}, 43 \%$ carbohydrate, $32 \%$ fat and

$63025 \%$ protein and was eaten over 10-15 minutes. Venous blood samples were drawn through a

631 stopcock in an antecubital vein immediately prior to breakfast, then at 30, 60, 120 and $180 \mathrm{~min}$ 
632 (just prior to the fMRI scan) then just after the scan at $300 \mathrm{~min}$ (Fig.1) to measure hormone levels

633 (see below). In addition, at each session, blood tests were conducted to measure $\mathrm{C}$ reactive protein,

634 ketones, cholesterol, triglyceride, and glycated hemoglobin (HbA1c) levels (Table S2). Participants

635 were confirmed to have negative blood ketones at each session.

636

637 Psychological, Physical Activity, Hunger Measures

638 Participants completed the Binge Eating Scale, the Dutch Eating Behavior (van Strien et al., 1986)

639 and Power of Food Questionnaires (Lowe et al., 2009) to assess the eating styles. Sensitivity to

640 reward and punishment was assessed using the Sensitivity to Punishment and Sensitivity to Reward

641 Questionnaire(Torrubia et al., 2001) and the BIS/BAS Scale (Carver and White, 1994),

642 depression levels using the Beck Depression Inventory(Beck A.T., 1972) ), and chronic stress

643 during the previous month using the Perceived Stress Scale (PSS) (Cohen et al., 1983) (Table S1).

644 Participants reported their activity levels over the past week by answering two questions: "Using the

645 past week a reference, how much time have you given to the following activities? Jogging, cycling,

646 swimming, cross-country skiing, aerobic dance or other similar activities." and "Over the past week,

647 how much time have you spent doing strenuous work, a physical activity or a sport other than

648 those mentioned in the previous question?" The mean scores from these two questions were used

649 to calculate changes in self-reported exercise (Table S2). Hunger levels were assessed using Visual

650 Analog Scales (VAS) four times throughout the day across the sessions (Fig. 1). On the VAS, we

651 asked the participants "On a scale from 0 to 10 how hungry do you feel now?" (Table S3).

652

653 
655 The blood samples were centrifuged, and plasma and serum stored at $-80 \mathrm{C}$ until analysis. All 656 samples from each participant were measured within the same assay. Plasma glucose was measured 657 by the glucose-oxidase technique (GM-9, Analox Instruments, Lunenberg, MA, USA), and insulin

658 using a specific radioimmunoassay (RIA) (Millipore, Billerica, MA, USA). We measured both 659 total and acylated ghrelin, as the latter is considered "active". Samples required acidification before 660 storage to prevent degradation (Hosoda and Kangawa, 2012). Both were measured by RIA 661 (Millipore, Billerica, MA, USA). Leptin was assayed using a solid phase ELISA (R\&D Systems 662 Inc, Minneapolis, MN, USA). The HOMA -IR (insulin resistance) index was calculated as fasting 663 (glucose $\mathrm{x}$ insulin)/22.5.

666 Neuroimaging was carried out with a Siemens Magnetom Trio 3T MRI scanner at the Montreal 667 Neurological Institute (MNI). High-resolution T1-weighted anatomical images with voxel size = $6681 \times 1 \times 1 \mathrm{~mm}$ were obtained first. Functional data were acquired with an echo-planar T2* weighted 669 sequence for BOLD contrast $\left(\mathrm{TR}=2 \mathrm{~s} ; \mathrm{TE}=30 \mathrm{~ms}\right.$; flip angle, $90^{\circ} ; \mathrm{FOV}=224 \mathrm{~mm}$, voxel size $=$ $670 \quad 3.5 \times 3.5 \times 3.5 \mathrm{~mm}^{3}$, number of slices $\left.=38\right)$.

671 For each session, participants underwent six 7-minute functional runs. During each run subjects 672 viewed images of food or scenery, presented via a projector and a mirror placed on the head coil. 673 The food and scenery images (examples in Fig.1) had been previously matched for visual appeal 674 (Malik et al., 2008). Each run comprised 36 unique images, 12 each of high and low-calorie foods 675 (e.g. brownies, vegetables) and scenery (Fig.1). The order of picture presentation was randomized 
676 across subjects and runs. Images were presented for 4.0 seconds and were followed by a rating of

677 the stimulus on a 1-4 scale. For food pictures, participants rated "How appetizing is this stimulus?"

678 and for scenery pictures participants rated "How pleasant is this stimulus?". Rating was followed by

679 a fixation cross with a jittered interstimulus interval (2.5-6.0 seconds). Stimulus presentation was

680 done using E-Prime (Psychology Software Tools Inc, Sharpsburg PA, USA). Ratings were entered

681 by subjects via a MR-compatible button device.

682

683 Quantification and Statistical Analysis

684 Hormone and Behavioral Analysis

685 The values and descriptive statistics for the hormone measurements are listed in Table S3. The

686 statistical analysis of hormonal and psychological measures was conducted using MATLAB

687 (Version R2015a, The MathWorks Inc., Natick, MA, USA). We log-transformed leptin and

688 insulin values to correct for non-normality. The longitudinal analyses were run using linear mixed

689 effects modelling (MATLAB function fitlme) with subject as a random effect. The Akaike

690 information criterion was utilized to select the best model (Table S4). To compare across sessions

691 and across time points, and to calculate the $\mathrm{F}$ and $\mathrm{p}$ values, we conducted linear hypothesis testing

692 on the linear mixed regression model coefficients using CoefTest implemented in MATLAB. The

693 graphs were produced using the raw data, but the statistical analyses reported were based on linear

694 mixed effect models. For correlations, we conducted Spearman rank correlations when the

695 distribution was not normal.

696

697 Imaging Data Analysis 
698 The T1-weighted MRIs were submitted to brain extraction using BEaST, a nonlocal segmentation

699 method applied to the images linearly registered to ICBM-MNI template (Eskildsen et al., 2012).

700 Pre-processing of the BOLD data was conducted using FEAT (FMRI Expert Analysis Tool,

701 Version 6, part of FMRIB's Software, www.fmrib.ox.ac.uk/fsl) and consisted of slice timing motion

702 correction, spatial smoothing $(6 \mathrm{~mm})$, and high-pass filtering with a cut-off frequency of $0.1 \mathrm{~Hz}$

703 (Jenkinson et al., 2012). Linear registration (6 parameters) to T1-weighted and non-linear

704 registration to standard T1-weighted ICBM-MNI152 template brain (voxel size $\left.=2 \times 2 \times 2 \mathrm{~mm}^{3}\right)$ were

705 completed using FLIRT (Jenkinson et al., 2002) and FNIRT (Andersson et al., 2007) respectively,

706 prior to statistical analysis.

709 For the first-level statistical analysis of the BOLD time-series, a general linear model (GLM) was

710 implemented using FILM (Woolrich et al., 2004). The regressors for the GLM were the motion

711 parameters, button presses for ratings, missed events, and the food and scenery image presentation

712 events which were modeled by convolving the time course with a double-gamma hemodynamic

713 response function (HRF) and applying temporal filtering. The resulting contrast images (i.e. food

714 minus scenery) were then passed onto a second-level fixed-effects analysis for each subject, to

715 obtain mean contrast estimates over runs within subjects by forcing random effects variance to zero

716 in FLAME (FMRIB's Local Analysis of Mixed Effects) (Beckmann et al., 2003; Woolrich et al.,

717 2004) The t-stat maps for the contrast of interest (i.e., session1 vs. session2, session2 vs. session3,

718 etc.) were combined across subjects for the third-level analysis. 
720 In the third level analysis, the results at the group level were analyzed using non-parametric

721 permutation tests $(\mathrm{n}=5,000$ permutations $)$ with the threshold-free cluster enhancement (TFCE)

722 algorithm using randomize in FSL (Smith and Nichols, 2009). In order to explore how the percent

723 weight loss or changes in hormone levels affected the reactivity to food cues (i.e. food vs. scenery

724 images), we included them as regressors in the GLM model. We utilized percent change in weight

725 loss to account for baseline weight.

727 To analyze the effects of weight loss on value related activity for food items, we performed a region

728 of interest analysis using a mask from a fMRI meta-analysis for subjective value at the time of

729 decision (see Fig.6A from (Bartra et al., 2013)). In addition, we investigated how weight loss related

730 to changes in BOLD in regions implicated in cognitive control. For this purpose, we restricted our

731 analysis to a regional forward-inference mask associated with the term "cognitive control" in the

732 NeuroSynth database of fMRI studies (http://neurosynth.org/analyses/terms/cognitive\%20control/)

733 (Yarkoni et al., 2011). Featquery from FSL was utilized to extract the percent change in mean beta

734 parameter estimates in each ROI that was used for plotting.

736 Functional Connectivity Analysis

737 For functional connectivity analysis we utilized psychophysiological interaction (PPI)(O’Reilly et

738 al., 2012). We selected a seed region based on the peak coordinate of food minus scenery at 739 session 1 minus session 2 (MNI: $\mathrm{x}=-10, \mathrm{y}=34, \mathrm{z}=-12)$. This peak was used to form a $4 \mathrm{~mm}$ 740 diameter seed region of interest (ROI) in the vmPFC. We further transformed this ROI in 
741 standard space into each individual's functional space using the previously obtained linear

742 transformations (from FLIRT). For each subject, we determined the peak voxel within the ROI,

743 and used it to create a new 4mm subject-specific ROI used to extract the mean BOLD time-series

744 signal. The mean time series of the seed ROI and the contrast of interest (i.e. food cues vs. scenery

745 images) were multiplied to create the PPI regressor in the first level analysis. Higher-level analysis

746 was repeated as stated above.

747 For all of the univariate results reported here, the significance of the clusters was determined by

748 threshold-free cluster enhancement (TFCE) with a significance level of $\mathrm{p}<0.05$ family wise error

749 rate (FWER). All coordinates are given in MNI space. 